\title{
Diagnosing the entropy budget of a climate model
}

\author{
By KLAUS FR AEDRICH ${ }^{1,2 *}$ and FR A N K LUN KEIT ${ }^{1}, \quad{ }^{1}$ Meteorologisches Institut, Universität Hamburg, \\ Bundesstr. 55, 20146 Hamburg, Germany; ${ }^{2}$ Max-Planck-Institut für Meteorologie, Bundesstr. 55, 20146 Hambursg, \\ Germany
}

(Manuscript received 26 October 2007; in final form 14 April 2008)

\begin{abstract}
A B STRACT
A general circulation model (GCM) of intermediate complexity (Planet Simulator) is subjected to an analysis of the entropy budget and its sensitivity. The entropy production is computed directly based on temperature and temperature tendencies and estimated indirectly based on boundary fluxes. For indirect estimates, the model shows reasonably good agreement with observations. The direct computation indicates deficits of the indirect measures, as they, for example, overestimate the material entropy production (that is, the production by turbulent fluxes). Sensitivity analyses of entropy production are provided, which, depending on changing parameters, hint to a possible applicability of maximum entropy production (MEP) under the constrained dynamics of a complex GCM.
\end{abstract}

\section{Introduction}

Climate model analysis is based on dynamical and statistical measures suitable for model-data intercomparison and validation. Traditionally, analyses concentrate on the terms contributing to the energy and momentum budgets of the climate system. Only a few analyses focus on entropy, although entropy budget investigations of the earth-atmosphere system have gained growing interest. More than half a century ago Lettau (1954) provided an early data analysis of the mass, energy and momentum budgets of the earth's atmospheric climate, supplemented by an estimate of the entropy balance. In the 1990s, the entropy subject has entered textbooks on the climate data analysis (Peixoto and Oort, 1992). However, when climate models are subjected to entropy budget analyses, this has been mainly confined to energy balance models. In particular, these simple systems have been used to demonstrate extremum principles at work (see Paltridge, 1975; Grassl, 1981; Lorenz et al., 2001 and others), with its steady-state dynamics following maximum entropy production (MEP, for one of the relevant reviews, see Ozawa et al., 2003, and some theoretical underpinning by Dewar, 2003).

If the MEP-principle is applicable in atmospheric or even climate dynamics, it may contribute substantially to climate model building and its performance evaluation: MEP of a simplified atmospheric global circulation model has been analysed to test MEP sensitivity by varying boundary layer (dissipation) properties with some success (Kleidon et al., 2003; 2006); this is also

\footnotetext{
* Corresponding author.

e-mail: klaus.fraedrich@zmaw.de

DOI: $10.1111 /$ j.1600-0870.2008.00338.x
}

the case for other dynamic parameters (Kunz et al., 2008) including the biosphere (Kleidon and Fraedrich, 2005). However, a recent appraisal (Goody, 2007) summarizes some of the problems related to MEP in a dynamically constrained environment.

Before the MEP-principle is tested in general circulation models (GCM) of the atmosphere or coupled atmosphere-ocean GCMs, their entropy budgets need to be evaluated. So far, complex GCMs' entropy budget has attracted less attention. One reason being that they 'are not generally constructed to ensure accurate treatment of entropy, but little is known about the significance of the resulting errors' (Woollings and Thuburn, 2006). Goody (2000) studies the entropy budget of the Goddard Institute of Space Studies (GISS) atmospheric GCM in comparison with data deduced from observations and values derived by Peixoto et al. (1991). Goody concludes that entropy inventories are not yet established to a desirable degree of accuracy, in particular regarding the sensitivity of model output to entropy errors, as for example the 'general coldness of climate models' (Johnson, 1997). The purpose of this paper is to subject a GCM of intermediate complexity to entropy budget analysis. Following Goody (2000), the entropy production is computed directly based on temperature and temperature tendencies and compared with indirect estimates based on boundary fluxes. Thus, uncertainties involved in the indirect measures obtained from observations or GCM simulations can be quantified. In addition, a sensitivity analysis of entropy production is provided, which, depending on changing parameters, may hint to the applicability of MEP in the constrained dynamics of complex GCMs.

The paper is structured as follows: the methods of entropy analysis are described and the circulation model Planet Simulator is introduced (Section 2). The entropy budget of the present 
day simulation is presented (Section 3) and its sensitivity (Section 4) is analysed changing continental and ocean properties: (a) desert versus woody continents and (b) the horizontal diffusivities of a purely diffusive ocean. A discussion concludes the analysis (Section 5).

\section{Methods, model and experimental design}

The Earth's climate is an open system, where energy is gained from the sun and radiated to space. These energy fluxes are associated with an import and an export of entropy. The difference between entropy exported to space and the received entropy flux from the sun is positive and defines the entropy production. Here and in the following, entropy productions are considered only if they are related to energy fluxes that enter the thermodynamic energy balance of the Earths climate system by diabatic heating. That is, entropy production by, for example, scattering is not taken into account.

With the net incoming solar radiation $S_{0}$, a brightness temperature of the sun $T_{\text {Sun }}$, the outgoing long-wave radiation $L_{0}$ at the top of the atmosphere and the brightness temperature of the earth $T_{\mathrm{E}}$, the surface area mean of the total entropy production $\mathrm{d} S / \mathrm{d} t$ is given by

$\frac{\mathrm{d} S}{\mathrm{~d} t}=\frac{L_{0}}{T_{\mathrm{E}}}-\frac{S_{0}}{T_{\text {Sun }}}$

(see, for example, Ozawa et al., 2003). With values of $240 \mathrm{~W} \mathrm{~m}^{-2}$ for $S_{0}$ and $L_{0}$ (that is, a climate in equilibrium) and assuming brightness temperatures $T_{\text {Sun }}$ and $T_{\mathrm{E}}$ of 5800 and $255 \mathrm{~K}$, respectively, the total entropy production is about $0.90 \mathrm{~W} \mathrm{~m}^{-2} \mathrm{~K}^{-1}$.

Various processes contribute to the entropy production: the absorption of short-wave radiation, the exchange of energy between the surface and the atmosphere by sensible and latent heat fluxes and by long-wave radiation plus internal diffusion and dissipation in the individual climate compartments. Following Ozawa et al. a simplified entropy budget of the Earth may be written as

$$
\begin{aligned}
\frac{\mathrm{d} S}{\mathrm{~d} t}= & \underbrace{\frac{L_{0}}{T_{\mathrm{E}}}-\frac{S_{0}}{T_{\mathrm{Sun}}}}_{0}=\underbrace{F_{\text {turb }}\left(\frac{1}{T_{\mathrm{A}}}-\frac{1}{T_{\mathrm{S}}}\right)}_{1}+\underbrace{F_{\mathrm{SWR} ; \mathrm{S}}\left(\frac{1}{T_{\mathrm{S}}}-\frac{1}{T_{\text {Sun }}}\right)}_{2} \\
& +\underbrace{F_{\text {SWR;A }}\left(\frac{1}{T_{\mathrm{A}}}-\frac{1}{T_{\text {Sun }}}\right)}_{3}+\underbrace{F_{\mathrm{LWR}}\left(\frac{1}{T_{\mathrm{A}}}-\frac{1}{T_{\mathrm{S}}}\right)}_{4} .
\end{aligned}
$$

The terms on the right hand side are the entropy productions by (1) turbulent fluxes ( $F_{\text {turb }}$; which are mainly latent and sensible heat fluxes); (2) the absorption of solar radiation at the surface $\left(F_{\text {SWR;S }}\right)$; (3) the absorption of solar radiation in the atmosphere $\left(F_{\text {SWR;A }}\right)$ and $(4)$ the interaction between atmosphere and surface by long-wave radiation $\left(F_{\mathrm{LWR}}\right) . T_{\mathrm{A}}$ and $T_{\mathrm{S}}$ denote 'effective' temperatures of the atmosphere and the surface, respectively, which are those temperatures at which the absorption/emission of the respective flux takes place. In the simplification used here, these temperatures are assumed to be the same for all processes.

Inserting observed fluxes $\left(L_{0}=S_{0}=240 \mathrm{~W} \mathrm{~m}^{-2}, F_{\text {turb }}=\right.$ $102 \mathrm{~W} \mathrm{~m}^{-2}, F_{\text {SWR } ; \mathrm{S}}=142 \mathrm{~W} \mathrm{~m}^{-2}, F_{\text {SWR;A }}=98 \mathrm{~W} \mathrm{~m}^{-2}, F_{\mathrm{LWR}}=$ $\left.40 \mathrm{~W} \mathrm{~m}^{-2}\right)$ and assuming effective temperatures $\left(T_{\mathrm{Sun}}=\right.$ $5800 \mathrm{~K}, T_{\mathrm{E}}=T_{\mathrm{A}}=255 \mathrm{~K}, T_{\mathrm{S}}=288 \mathrm{~K}$ ), the contributions to the total entropy production (0) are (see fig. 5 in Ozawa et al.; in $\left.\mathrm{W} \mathrm{m}^{-2} \mathrm{~K}^{-1}\right)$ :

(0) 0.900 [100\%]; (1) 0.046 [5\%]; (2) 0.489 [52\%];

(3) $0.367[41 \%]$; (4) 0.018 [2\%]

or, using the same temperatures but observed fluxes from Kiehl and Trenberth (1997; with $L_{0}=S_{0}=235 \mathrm{~W} \mathrm{~m}^{-2}, F_{\text {turb }}=$ $102 \mathrm{~W} \mathrm{~m}^{-2}, F_{\mathrm{SWR} ; \mathrm{S}}=168 \mathrm{~W} \mathrm{~m}^{-2}, F_{\mathrm{SWR} ; \mathrm{A}}=67 \mathrm{~W} \mathrm{~m}^{-2}, F_{\mathrm{LWR}}=$ $66 \mathrm{~W} \mathrm{~m}^{-2}$ ):

(0) 0.881 [100\%]; (1) 0.046 [5\%]; (2) 0.554 [63\%];

(3) 0.251 [29\%]; (4) 0.030 [3\%],

which, in comparison with Ozawa et al., estimate a larger contribution by the absorption of short-wave radiation at the surface, at the expense of the absorption in the atmosphere. Also, the atmosphere-surface interaction due to long-wave radiation is larger and contributes more to the total (which, however, is still a very small value).

Although radiative processes dominate the entropy production, the turbulent fluxes, or the material entropy production, are supposed to be the subject of the maximization principle (Weiss, 1996; Ozawa et al., 2003). Goody (2000) argued that radiative entropy productions cancel each other and, thus, have no relevance for the system and could be omitted.

Besides computing the material entropy production directly, it may indirectly be determined by the radiative processes alone. Thus, reorganizing eq. (2) gives

$$
\begin{aligned}
F_{\text {turb }}\left(\frac{1}{T_{\mathrm{A}}}-\frac{1}{T_{\mathrm{S}}}\right)= & F_{\mathrm{SWR} ; \mathrm{S}}\left(\frac{1}{T_{\mathrm{S}}}-\frac{1}{T_{\mathrm{Sun}}}\right) \\
& +F_{\mathrm{SWR} ; \mathrm{A}}\left(\frac{1}{T_{\mathrm{A}}}-\frac{1}{T_{\mathrm{Sun}}}\right) \\
& +F_{\mathrm{LWR}}\left(\frac{1}{T_{\mathrm{A}}}-\frac{1}{T_{\mathrm{S}}}\right) \\
& -\frac{L_{0}}{T_{\mathrm{E}}}-\frac{S_{0}}{T_{\mathrm{Sun}}} .
\end{aligned}
$$

However, it is not clear how accurate the estimates above are since they involve a couple of assumptions and simplifications; for example: the use of annual and global mean fluxes, the disregard of diffusion and dissipation within one subsystem and the choice of effective temperatures. These are only more or less well-founded approximations, since they represent a combination of all temperatures at which the respective processes take place. Unfortunately, a more precise estimate is almost impossible with the data available. 


\subsection{Model}

A global climate model is a surrogate for the real world and allows to compute the entropy budget directly and to compare the results with the indirect estimates. The Planet Simulator (Fraedrich et al., 2005a,b) is a climate model of intermediate complexity. The atmospheric dynamic is modelled using the primitive equations formulated for vorticity, divergence, temperature and the logarithm of surface pressure. These equations are solved using the spectral transform method (Eliasen et al., 1970; Orszag, 1970). Unresolved processes are parametrized. The parametrization packet consists of long- (Sasamori, 1968) and short- (Lacis and Hansen, 1974) wave radiation, interactive clouds (Stephens, 1978; Stephens et al., 1984; Slingo and Slingo, 1991), moist (Kuo, 1965; 1974) and dry convection, large-scale precipitation, boundary layer fluxes of latent and sensible heat and vertical and horizontal diffusion (Louis, 1979; Louis et al., 1982; Laursen and Eliasen, 1989; Roeckner et al., 1992). The land surface scheme uses five diffusive layers for the temperature and a bucket model for the soil hydrology. The oceanic part is a $50 \mathrm{~m}$ mixed-layer (swamp) ocean, which includes a 0-dimensional thermodynamic sea ice model. For the ocean, the horizontal transport of heat can either be prescribed or parametrized by horizontal diffusion. The model is freely available under http://www.mi.uni-hamburg.de/plasim.

Here the Planet Simulator is used in T21 horizontal resolution (approx. $5.6^{\circ} \times 5.6^{\circ}$ on the corresponding Gaussian grid) with 10 not equally spaced sigma levels in the vertical. The Planet Simulator climate of the present day simulation is reasonable, considering the coarse resolution and relative simplicity of the model compared with a state-of-the-art GCM as demonstrated by the annual mean surface pressure, temperature and precipitation (and the respective zonal averages, Fig. 1). Also, the globally averaged energy fluxes are in good agreement with observations (Fig. 2). Larger differences (more than 10\%) can be noted in the sensible and latent heat flux where the Planet Simulator shows lower (sensible) and higher (latent) heat fluxes than observed. In addition, an imbalance of about $+2 \mathrm{~W} \mathrm{~m}^{-2}$ exists at the top of the model atmosphere. This imbalance is mostly due to conversion of potential to kinetic energy, since the kinetic energy loss by friction is not transferred back to the energy cycle (by heating of the environment). In the present simulations, the model is tuned in a way that this imbalance is present at the top of the atmosphere while the sum of all fluxes vanishes at the surface (that is, atmosphere and ocean are assumed to be in equilibrium with each other).

\subsection{Entropy budget and experimental design}

In the Planet Simulator the contribution $(\mathrm{d} S / \mathrm{d} t)_{j, g}$ from each individual process $j$ (e.g. long-wave radiation) to the total entropy production is 'directly' computed for each grid box $g$ using the respective local heat capacity $c p$, temperature $T$ and temperature
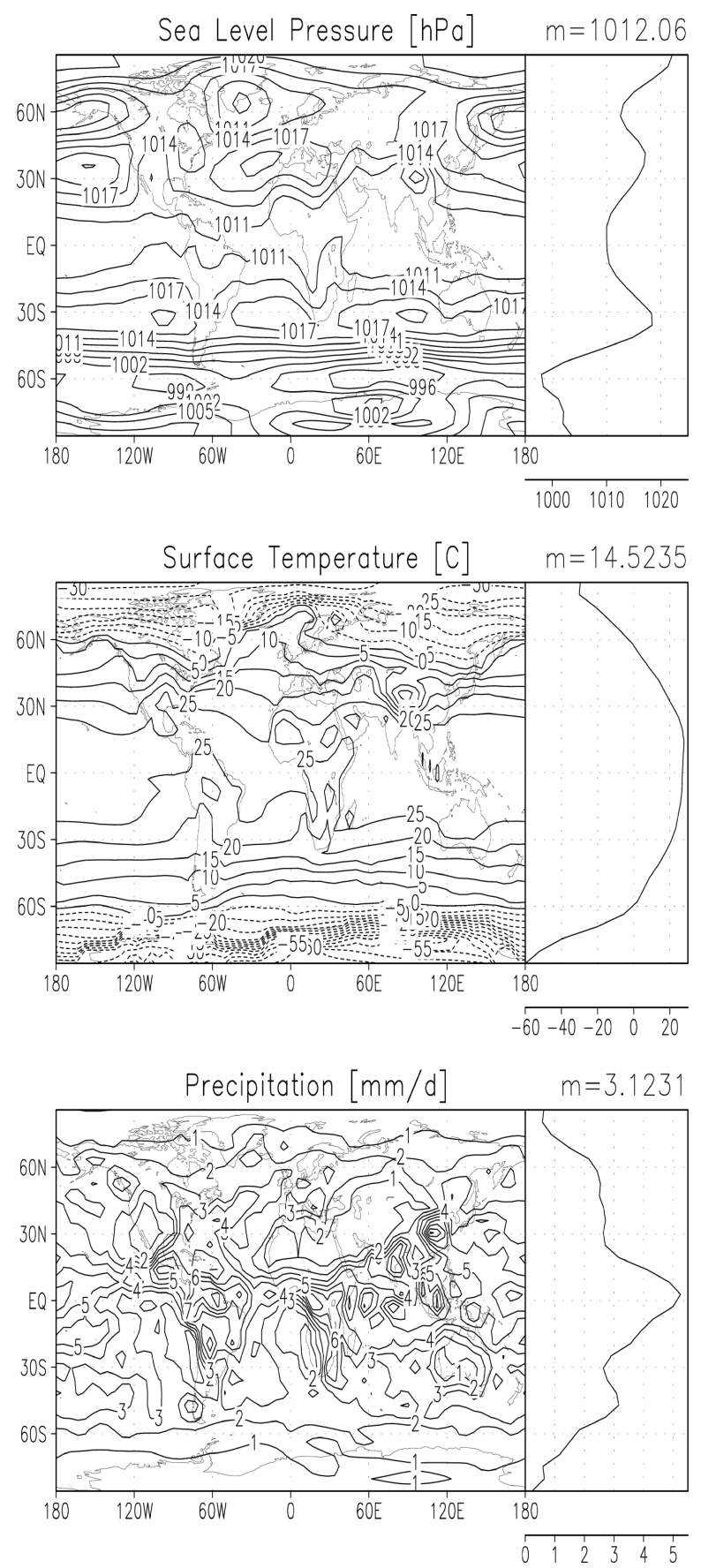

Fig. 1. Horizontal distributions and zonal averages of the annual mean sea level pressure (top panel; in $\mathrm{hPa}$ ), surface temperature (middle panel; in ${ }^{\circ} \mathrm{C}$ ) and precipitation (bottom panel; in $\mathrm{mm} \mathrm{d}^{-1}$ ) for the present day simulation.

tendency $(\partial T / \partial t)_{j}$ :

$\left(\frac{\mathrm{d} S}{\mathrm{~d} t}\right)_{j, g}=c p \frac{1}{T}\left(\frac{\partial T}{\partial t}\right)_{j} \mathrm{~d} M$.

$(\partial T / \partial t)_{j}$ denotes that fraction of the total temperature change that is caused by process $j . \mathrm{d} M$ is the mass related to the respective 


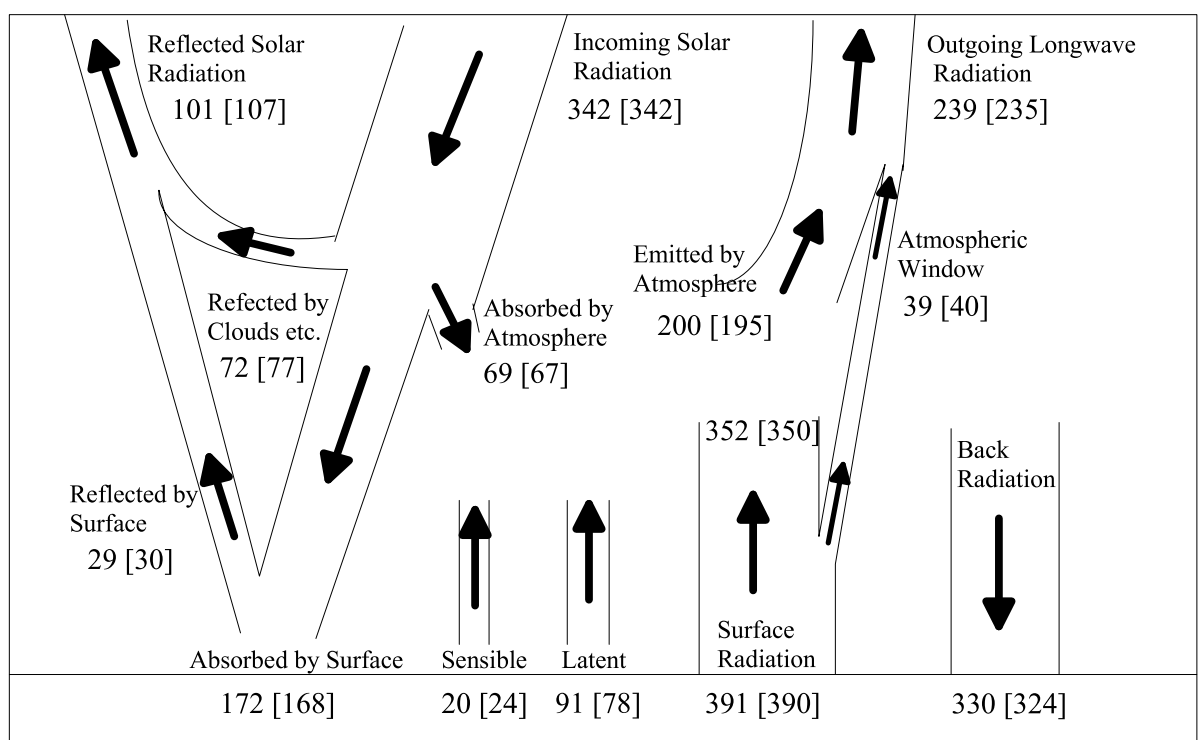

Fig. 2. The annual global mean energy budget (in $\mathrm{W} \mathrm{m}^{-2}$ ) for the present day simulation and, in brackets, for observations (after Kiehl and Trenberth, 1997).

grid box. The sum of $(\mathrm{d} S / \mathrm{d} t)_{j, g}$ over all processes $j$ at one grid box defines the contribution of that grid box to the global entropy production. The total entropy production of the system $\mathrm{d} S / \mathrm{d} t$ is the sum over all grid boxes and may be partitioned into an atmospheric and a surface part, where the latter is dominated by the ocean.

Many processes can contribute to the entropy budget (see, e.g. Goody, 2000), but not all of them are present in the Planet Simulator. Here, entropy can be produced by short- and longwave radiation, surface sensible and latent heat fluxes, the release of latent heat by condensation, vertical and horizontal diffusion, dry convection and the spectral dynamical core, as described in Woollings and Thuburn (2006). Also, the oceanic heat transport in the swamp ocean (either prescribed or modelled by horizontal diffusion) can produce entropy. In addition, the dissipation of kinetic energy by friction is in principle a source of entropy. However, since in the model the loss by friction is not transferred back by heating, this contribution is considered as part of the residuum.

Using the Planet Simulator, the following questions on entropy production of the climate system are addressed. The related sets of simulations consist of 75 simulated years of which the last $25 \mathrm{yr}$ are subjected to the entropy budget analysis.

(1) Entropy diagnostics and validation: Which processes contribute how much to the global entropy production in a present day simulation (in comparison to observations)? How exact are the 'indirect' entropy production estimates by knowing the global averaged fluxes at the boundaries only (or, in other words, which are the 'effective' temperatures in eq. 2) when comparing them with direct computations using temperature and temperature tendencies? Present day conditions for solar radiation, greenhouse gas content, vegetation, etc. define the set up for the simulation. The ocean is represented by a $50 \mathrm{~m}$ swamp (including a 0-dimensional thermodynamic sea ice model) where the oceanic heat transport is prescribed to assure an observed climatological mean sea surface temperature and sea ice distribution.

(2) Sensitivity analyses: How much do the global entropy production and the individual contributions change with modified boundary conditions, that is, different climate conditions? Here, the sensitivity to vegetation extremes and to the oceanic heat transport are investigated. To some extent the results are related to the validity of the concept of MEP and may give some hint in this respect. Note, however, that it is not our aim to verify the MEP-concept nor is the set up thought to be appropriate to do so. To estimate the sensitivity of the entropy production to changes in boundary conditions two experimental setups are chosen: (a) The effect of vegetation extremes is assessed in simulations similar to Fraedrich et al. (2005b). Here, global desert and global forest boundary conditions for land surfaces are prescribed. An interactive mixed layer ocean with prescribed heat transport equal to the present day simulation is used. (b) The sensitivity to the oceanic heat transport is investigated in a set of simulations similar to (1) but with oceanic transport modelled by horizontal diffusion using different diffusivities.

\section{Entropy diagnostics: present day conditions}

The directly computed entropy productions indicate that only the absorption of solar radiation, the sensible and latent heat fluxes (i.e. for the atmosphere, the release of latent heat by condensation) and the long-wave radiation contribute significantly to the global entropy budget. The other processes (vertical 
Table 1. Contributions of different processes to the global entropy balance (in $\mathrm{W} \mathrm{m}^{-2} \mathrm{~K}^{-1}$ ) in the present day run

\begin{tabular}{lccc}
\hline Process & Atmosphere & Surface & Total \\
\hline Absorption of sw radition & 0.267 & 0.587 & 0.854 \\
Emission of lw radiation to space & -0.781 & -0.140 & -0.921 \\
Atm-sfc interaction by lw radiation & 0.078 & -0.072 & 0.006 \\
Internal interaction by lw radiation & 0.028 & - & 0.028 \\
Sensible heat flux & 0.070 & -0.069 & 0.001 \\
Latent heat flux & 0.334 & -0.306 & 0.028 \\
Kinetic energy dissipation & 0.006 & - & 0.006 \\
\hline
\end{tabular}

Only significant contributions are listed. Note that the contribution by kinetic energy dissipation (friction) is only potential since the related energy is not used to heat the environment.

and horizontal diffusion, dry convection, the dynamics and the prescribed heat transport in the ocean) do not contribute more than the residuum, which is taken as a measure of uncertainty. This residuum is of the order of some $10^{-3} \mathrm{~W} \mathrm{~m}^{-2} \mathrm{~K}^{-1}$ and mainly caused by the imbalance of the fluxes at the top of the atmosphere (that is the conversion of potential to kinetic energy; see above), internal variability and the numerics (for example, the artificial fix of the global mass, which is common for spectral models). In addition, it should be noted that the numerical uncertainty of the global entropy production terms (due the summation of large positive and negative contributions to obtain a small global mean) appears to be about $0.001 \mathrm{~W} \mathrm{~m}^{-2} \mathrm{~K}^{-1}$. The contribution by long-wave radiation is divided into three parts: the export into space (from atmosphere and surface), which (together with the solar input) defines the total entropy production (see eq. (1)), the interaction between surface and atmosphere and the interactions between the atmospheric levels.

The direct contributions by surface and atmospheric processes are shown in Table 1. To allow a better comparison with Goody (2000; his tables 3, 5, 6 and 7), a potential contribution by kinetic energy dissipation (friction) to the residuum is also included assuming that the environment is heated instantaneously. For the surface, entropy is produced by the absorption of solar radiation. Sensible and latent heat fluxes diminish the entropy of the surface by export to the atmosphere. Also, the atmosphere-surface interaction by long-wave radiation results in a net entropy loss for the surface. Subtracting the entropy import from the sun (the net surface short-wave radiation divided by the suns temperature) by the export of entropy to space through the atmospheric window, one obtains the contribution of the surface to the total entropy production of the climate system, which is $0.11 \mathrm{~W} \mathrm{~m}^{-2} \mathrm{~K}^{-1}$. Note that the prescribed oceanic heat transport, although comparable with observations (see Fig. 4), does not produce a significant amount of entropy (less than $0.001 \mathrm{~W} \mathrm{~m}^{-2} \mathrm{~K}^{-1}$ ) consistent with the estimates by Weiss (1996).
In the atmosphere, the largest contribution comes from the release of latent heat, followed by the absorption of solar radiation. The productions by sensible heat flux and by the long-wave radiation interacting with the surface are of same order. The interaction between the atmospheric levels by long-wave radiation is the smallest significant contribution. With the export to space and the import from the sun, the atmospheric contribution to the total entropy production is $0.77 \mathrm{~W} \mathrm{~m}^{-2} \mathrm{~K}^{-1}$.

The difference between the gain of entropy in the atmosphere and its loss at the surface due to latent and sensible heat fluxes, define the material entropy production (internal dissipation and diffusion are not significant). With $0.029 \mathrm{~W} \mathrm{~m}^{-2} \mathrm{~K}^{-1}$, the material entropy production contributes $3 \%$ to the total entropy production $\left(0.88 \mathrm{~W} \mathrm{~m}^{-2} \mathrm{~K}^{-1}\right)$. Deriving also terms (2) to (4) in the balance estimate (eq. (2)) from the direct measures yields:

(0) $0.880[100 \%]$; (1) 0.029 [3\%]; (2) 0.557 [63\%];

(3) $0.255[29 \%]$; (4) $0.007[1 \%]$

This balance needs to be closed by the internal atmospheric level-to-level interaction by long-wave radiation $(0.028$ $\mathrm{W} \mathrm{m}^{-2} \mathrm{~K}^{-1}$ [3\%]), which is not considered in eq. (2), and by the residuum $\left(0.007 \mathrm{~W} \mathrm{~m}^{-2} \mathrm{~K}^{-1}[1 \%]\right.$, where $0.006 \mathrm{~W} \mathrm{~m}^{-2} \mathrm{~K}^{-1}$ may be attributed to kinetic energy dissipation, see above).

The indirect estimates are derived for comparison by the fluxes at the boundaries alone, using the same assumption for effective temperatures $\left(T_{\mathrm{A}}=T_{\mathrm{E}}=255 \mathrm{~K}, T_{\mathrm{S}}=288 \mathrm{~K}\right)$ as for the observations. The respective numbers for the terms (0) to (4) are:

(0) $0.903[100 \%$; (1) $0.050[6 \%$; (2) 0.567 [63\%];

(3) 0.259 [29\%]; (4) $0.027[3 \%]$.

Here, the balance is closed by adding a value of 0.008 [1\%] to (0) assuming that the thermodynamic energy gain by friction is directly radiated into space at temperature $T_{\mathrm{A}}$. Considering the good agreement of the boundary fluxes with the observed ones, the overall agreement of the entropy balance is unsurprising. However, the larger difference for the material production is worth mentioning since, following the arguments by Goody (2000) and others, this production is most important. Here, the material production consists of two terms: the sensible heat flux and the production by moist processes (latent heat flux and condensation), which contribute 0.009 and $0.041 \mathrm{~W} \mathrm{~m}^{-2} \mathrm{~K}^{-1}$, respectively, compared with 0.011 and $0.035 \mathrm{~W} \mathrm{~m}^{-2} \mathrm{~K}^{-1}$ for the observations from Kiehl and Trenberth (1997). Using a different method, Goody estimates values of about 0.002 and $0.019 \mathrm{~W} \mathrm{~m}^{-2} \mathrm{~K}^{-1}$, respectively, in relatively close agreement to Peixoto et al. $\left(0.002\right.$ and $\left.0.023 \mathrm{~W} \mathrm{~m}^{-2} \mathrm{~K}^{-1}\right)$. In principle, also the production by heating due to momentum dissipation would enter the material production. Goody and Peixoto et al. (1991) estimate values of about 0.011 and $0.007(0.009) \mathrm{W} \mathrm{m}^{-2} \mathrm{~K}^{-1}$ for this contribution, which result in considerably lower total material entropy productions of 0.033 and $0.031(0.034) \mathrm{W} \mathrm{m}^{-2} \mathrm{~K}^{-1}$. 
As noted above, we do not consider the momentum dissipation since it does not enter the diabatic heating in the model. In addition, it also does not appear in the observed energy budget. The residuum of $0.008 \mathrm{~W} \mathrm{~m}^{-2} \mathrm{~K}^{-1}$ as an estimate for the momentum dissipation is in good agreement with Goody and Peixoto et al., but would further increase the differences between the total material productions. However, including the gain of heat by momentum dissipation in the model will affect all other terms too, and it is not obvious which balance will finally be obtained.

The indirect estimate of the material entropy production also comprises large errors compared with the direct measures, which fit better to the estimates by Goody and Peixoto et al. Also, the atmosphere-surface interaction by long-wave radiation (4) is substantially different. An error of $0.021 \mathrm{~W} \mathrm{~m}^{-2} \mathrm{~K}^{-1}$ [42\%] is present for the material entropy production. Since the average fluxes entering the calculations are the same in both cases, the effective temperatures for the indirect estimate seem not to be adequately chosen. The long-wave interaction between surface and atmosphere is only marginal in the direct measure (of the same order as the residuum), contributing 3\% in the estimates. There are two reasons for this: first, about $39 \mathrm{~W} \mathrm{~m}^{-2}$ of the surface long-wave radiation is directly transmitted into space through the atmospheric window, which is not considered in the estimate. Second, most of the long-wave interaction with the surface takes place at lower levels where the atmospheric temperature is closer to the value at the surface than the value used in the estimate; that is, in this case also, $T_{\mathrm{A}}$ and $T_{\mathrm{S}}$ may not have been chosen adequately.

A better match between the real and the estimated entropy balance may be obtained by introducing different effective temperatures and by including the contribution of the internal interaction by long-wave radiation. Since the flux used by the latter process cannot be assessed based on the fluxes at the boundaries only, its contribution is represented by a residuum ( $\left.\mathrm{d} S_{\mathrm{LWR}}\right)$. In the new estimate, each process is related to a different effective atmospheric or surface temperature:

$$
\begin{aligned}
& \frac{\mathrm{d} S}{\mathrm{~d} t}=\underbrace{\frac{L_{0}}{T_{\mathrm{E}}}-\frac{S_{0}}{T_{\mathrm{Sun}}}}_{0}=\underbrace{F_{\text {turb }}\left(\frac{1}{T_{\mathrm{A} 1}}-\frac{1}{T_{\mathrm{S} 1}}\right)}_{1} \\
& +\underbrace{F_{\mathrm{SWR} ; \mathrm{S}}\left(\frac{1}{T_{\mathrm{S} 2}}-\frac{1}{T_{\mathrm{Sun}}}\right)}_{2} \\
& +\underbrace{F_{\text {SWR;A }}\left(\frac{1}{T_{\mathrm{A} 2}}-\frac{1}{T_{\text {Sun }}}\right)}_{3} \\
& +\underbrace{F_{\mathrm{LWR}}\left(\frac{1}{T_{\mathrm{A} 3}}-\frac{1}{T_{\mathrm{S} 3}}\right)}_{4}+\mathrm{d} S_{\mathrm{LWR}} .
\end{aligned}
$$

Of course, in the model case, a perfect match can be archived when the effective temperatures are computed from the direct measures and fluxes (see Fig. 2 and Table 1), which leads to the following effective temperatures (in $\mathrm{K}$, rounded):

$$
\begin{gathered}
T_{\mathrm{E}}=272, T_{\mathrm{A} 1}=276, T_{\mathrm{S} 1}=291, T_{\mathrm{A} 2}=258, \\
T_{\mathrm{S} 2}=294, T_{\mathrm{A} 3}=285, T_{\mathrm{S} 3}=291 .
\end{gathered}
$$

Since the complete entropy production by long-wave surfaceatmosphere interaction is as small as the uncertainty, $T_{\mathrm{A} 3}$ and $T_{\mathrm{S} 3}$ are computed from the surface to atmosphere components only (which are large and give a robust estimate). Given these temperatures together with the observed fluxes (Kiehl and Trenberth, 1997) and accounting for the atmospheric window for $F_{\text {LWR }}$ (i.e. setting $F_{\text {LWR }}$ to $26 \mathrm{~W} \mathrm{~m}^{-2}$ ), the entropy balance terms are (in $\mathrm{W} \mathrm{m}^{-2} \mathrm{~K}^{-1}$ )
(0) 0.823 [100\%]; (1) 0.019 [2\%]; (2) 0.542 [66\%];

(3) $0.248[30 \%]$; (4) $0.002[<1 \%]$

The residuum (which may be taken as an estimate of the internal long-wave interaction) is $0.012 \mathrm{~W} \mathrm{~m}^{-2} \mathrm{~K}^{-1}$ [1\%]. This new estimate provides an even smaller material entropy production, which is closer to the estimates by Goody and Peixoto et al.

In summarizing, the model agrees reasonably well with observations regarding the indirect measures of entropy production used here. However, the largest differences (about 10\%) occur for the material production (1), which is supposed to be most important, in particular regarding the MEP principle. Differences to material entropy productions estimated by Goody and Peixoto et al. are even larger. Here, neglecting entropy production by momentum dissipation, appears also to be crucial.

\section{Sensitivity analyses: vegetation extremes and diffusive ocean}

The sensitivity of the model's entropy production to changes in boundary conditions is investigated in two sets of experiments: (1) the sensitivity to vegetation is analysed in two experiments in which the land surface is prescribed to be either totally covered by vegetation (rain forest) or by desert; (2) the sensitivity to the oceanic heat transport is studied by including horizontal temperature diffusion in the swamp ocean, varying the diffusivity.

\subsection{Vegetation extremes}

The two simulations analysed here are similar to the 'green planet' and 'desert world' experiments, estimating the effect of vegetation extremes on the atmospheric circulation (Fraedrich et al., 2005b). The experimental setup is adopted from Fraedrich et al. (1999) and Kleidon et al. (2000). Here, the simulations including the mixed-layer ocean (with prescribed present day oceanic transport) are chosen because they show, due to atmosphereocean interaction, a larger response than the uncoupled atmospheric model runs, and the coupling ensures balanced heat fluxes at the surface. 


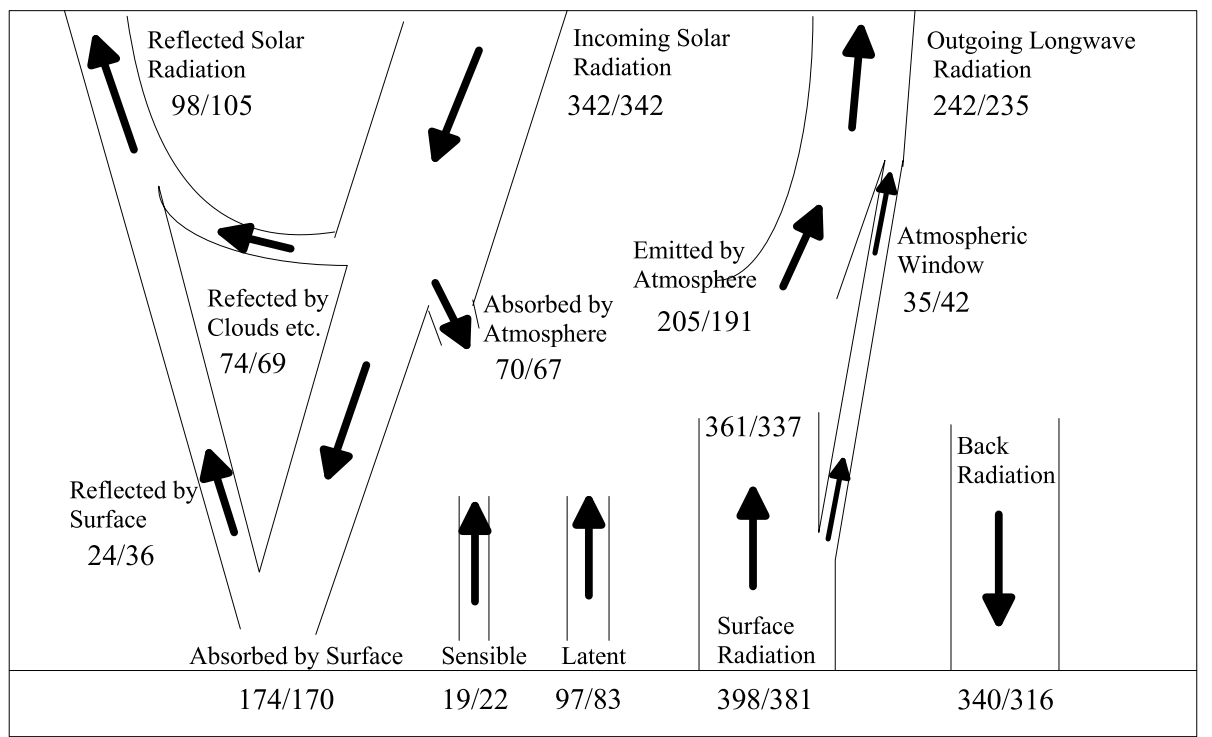

Fig. 3. The annual global mean energy budget (in $\mathrm{W} \mathrm{m}^{-2}$ ) for the green planet and for the desert world simulation (green/desert).

Significant differences between the climates of the green planet and the desert world occur in the energy and the water cycle. A description of the model setup and an extensive discussion of the results can be found in Fraedrich et al. (2005b). Figure 3 presents the global energy balance for both simulations. The following differences can be noted: (1) The planetary albedo is about $7 \%$ lower on the green planet. (2) The enhanced water cycle on the green planet is reflected by the larger latent heat flux (about $12 \mathrm{~W} \mathrm{~m}^{-2} ; 17 \%$ ). (3) Due to the enhanced atmospheric water vapour content, the atmospheric long-wave emission/absorption is enhanced, leading to an increase of the outgoing long-wave radiation by about $7 \mathrm{~W} \mathrm{~m}^{-2}(3 \%)$.

Table 2 gives the direct measures of entropy production in the two simulations. Due to the lower planetary albedo (that is, more short-wave input resulting in more long-wave emission) in the green planet, the total entropy production is about 0.02 $\mathrm{W} \mathrm{m}{ }^{-2} \mathrm{~K}^{-1}(2 \%)$ larger than in the desert world. The increase of entropy production is present in all parts with the largest contribution from the absorption of short-wave radiation. The material entropy production is $0.005 \mathrm{~W} \mathrm{~m}^{-2} \mathrm{~K}^{-1}(19 \%)$ larger in the green planet. It is noteworthy that the entropy production in the green planet simulation exceeds that of the present day by about $1 \%$ for the total and $11 \%$ for the material production. These results suggest that, regarding the MEP principle, a green planet would be the preferred state of the climate system. However, constraints for plant growth, like water availability and sensitivity to frost temperature, limit the applicability of this concept.

\subsection{Oceanic heat transport}

To investigate the sensitivity of the entropy production to the oceanic heat transport, a set of six simulations is performed. The setup of these simulations is similar to the present day run except that the oceanic heat transport is not prescribed but modelled by horizontal temperature diffusion. The value of the horizontal diffusivity $k_{\text {oce }}$ defines the member of the set. Simulations are performed for $k_{\text {oce }}=0,10^{3}, 10^{4}, 10^{5}, 10^{6}$ and $10^{7} \mathrm{~m}^{2} \mathrm{~s}^{-1}$.

Due to the increase of the oceanic heat transport, the equator to pole temperature gradient decreases with increasing diffusivity. Figure 4 shows the annual mean heat transports (oceanic

Table 2. As Table 1 but for the green planet and desert world simulations

\begin{tabular}{lccc}
\hline Process & Atmosphere green/desert & Surface green/desert & Total green/desert \\
\hline Absorption of sw radiation & $0.271 / 0.262$ & $0.589 / 0.581$ & $0.860 / 0.843$ \\
Emission of lw radiation to space & $-0.800 /-0.755$ & $-0.130 /-0.154$ & $-0.930 /-0.909$ \\
Atm-sfc interaction by lw radiation & $0.074 / 0.080$ & $-0.068 /-0.073$ & $0.006 / 0.007$ \\
Internal interaction by lw radiation & $0.029 / 0.027$ & - & $0.029 / 0.027$ \\
Sensible heat flux & $0.066 / 0.074$ & $-0.064 /-0.073$ & $0.002 / 0.001$ \\
Latent heat flux & $0.356 / 0.306$ & $-0.327 /-0.281$ & $0.029 / 0.025$ \\
\hline
\end{tabular}



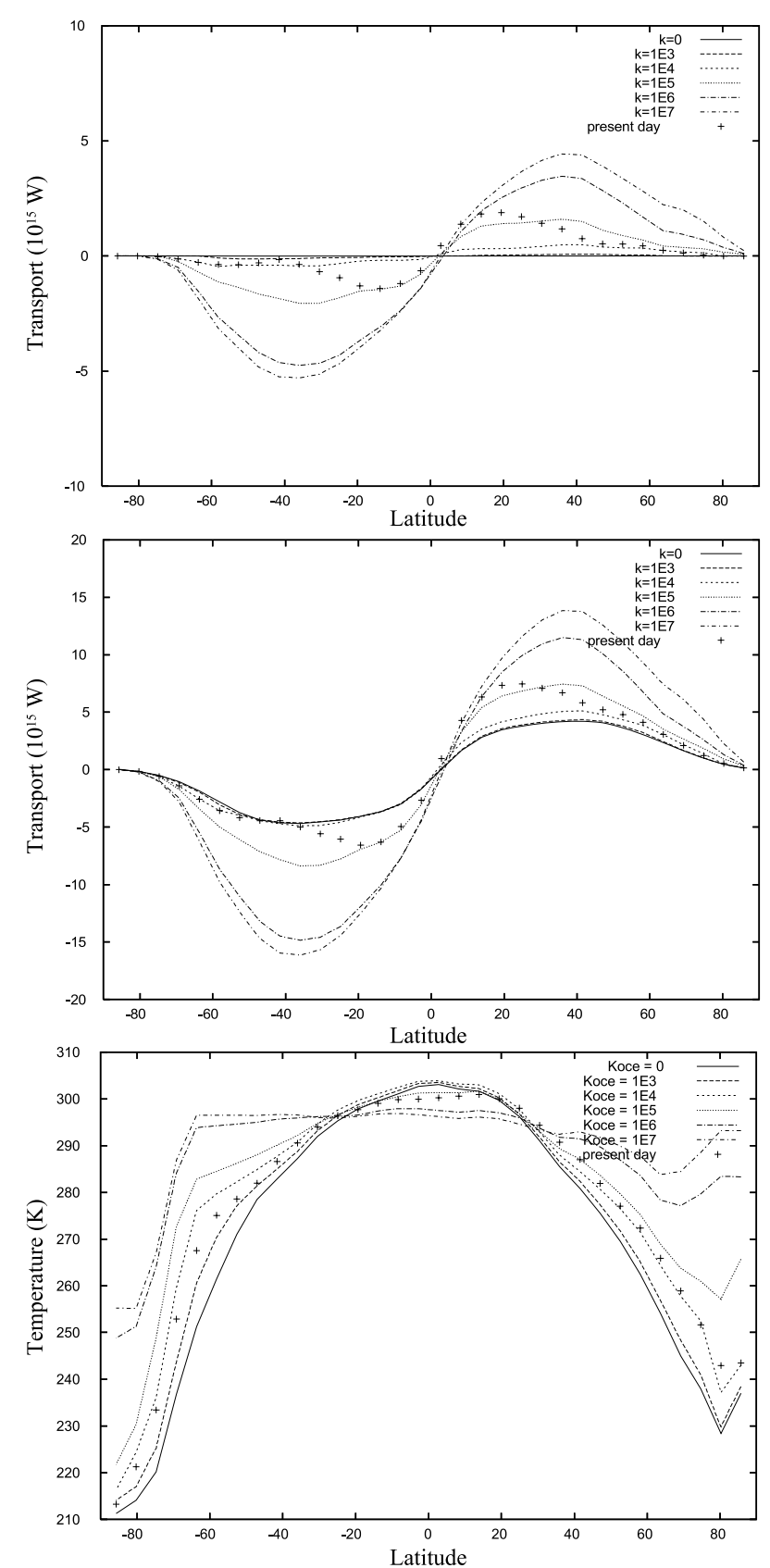

Fig. 4. Oceanic (top panel) and annual mean total (middle panel) heat transport (in $10^{15} \mathrm{~W}$ ) and zonally averaged surface temperature (bottom panel; in $\mathrm{K}$ ) for all $k_{\text {oce }}$ and for the present day simulation.

and total) and the zonally averaged surface temperatures for all simulations including the present day reference run (see Section $3)$. Simulations with $k_{\text {oce }}=0$ and $10^{3} \mathrm{~m}^{2} \mathrm{~s}^{-1}$ show a stronger temperature gradient than the present day simulation with colder temperatures in high latitudes and, therefore, larger sea ice extent. Sea ice is totally absent in the $k_{\text {oce }}=10^{6}$ and $10^{7} \mathrm{~m}^{2} \mathrm{~s}^{-1}$ simulations when the oceanic temperature is nearly homoge- neous. The drastic change of the lower boundary is associated with strong changes of the atmospheric circulation. For example, Fig. 5 shows the distribution of the annual mean precipitation for simulation $k_{\text {oce }}=0$ and $10^{7} \mathrm{~m}^{2} \mathrm{~s}^{-1}$. Whereas for $k_{\text {oce }}=0 \mathrm{~m}^{2} \mathrm{~s}^{-1}$ a sharp ITCZ exists, there is no ITCZ in the $k_{\text {oce }}=10^{7} \mathrm{~m}^{2} \mathrm{~s}^{-1}$ case with a slightly enhanced global average precipitation.

Table 3 lists annually and globally averaged fluxes and entropy productions from all members of the experiment. It is important to note that the contribution to the entropy production by temperature diffusion in the ocean is only barely significant (with maximum values of the order of $1.510^{-3} \mathrm{~W} \mathrm{~m}^{-2} \mathrm{~K}^{-1}$ for $k_{\text {oce }}=$ $10^{5}, 10^{6}$ and $\left.10^{7} \mathrm{~m}^{2} \mathrm{~s}^{-1}\right)$. Moreover, there is no distinct maximum notable for one of the diffusivities. Regarding the pronounced differences demonstrated in Figs. 4 and 5, the differences in the global fluxes and entropy productions are relatively small. The following results can be noted: (1) The reduction of sea ice (and snow cover) due to larger oceanic heat transport is related to a decrease of the planetary albedo (the cloud albedo, though increasing, does not compensate the ice/snow effect). (2) The net incoming solar radiation increases from about $239 \mathrm{~W} \mathrm{~m}^{-2}$ for $k_{\text {oce }}=0 \mathrm{~m}^{2} \mathrm{~s}^{-1}$ to about $246 \mathrm{~W} \mathrm{~m}^{-2}$ for $k_{\text {oce }}=10^{7} \mathrm{~m}^{2} \mathrm{~s}^{-1}$, accompanied by a related increase of the outgoing long-wave radiation.

The surface turbulent heat fluxes (sensible and latent heat fluxes) show an increase of the latent heat flux and a maximum for the sensible heat flux for $k_{\text {oce }}=10^{4} \mathrm{~m}^{2} \mathrm{~s}^{-1}$. In this case, also the total turbulent heat flux has a maximum. This suggests that, regarding the estimate of entropy production terms according to eq. (2), the material entropy production also has its maximum for $k_{\text {oce }}=10^{4} \mathrm{~m}^{2} \mathrm{~s}^{-1}$. This result fits to the MEP principle since the $k_{\text {oce }}=10^{4} \mathrm{~m}^{2} \mathrm{~s}^{-1}$ case is close to the present day climate (in terms of the heat transport and the zonally averaged surface temperature; see Fig. 4), although $k_{\text {oce }}=10^{5} \mathrm{~m}^{2} \mathrm{~s}^{-1}$ leads to a better agreement. Indeed, a pronounced maximum of the direct measured material entropy production (the sum of the productions by sensible and latent heat fluxes) for the atmosphere is found for $k_{\text {oce }}=10^{4} \mathrm{~m}^{2} \mathrm{~s}^{-1}\left(0.008 \mathrm{~W} \mathrm{~m}^{-2} \mathrm{~K}^{-1}\right.$ larger than the next $)$. For the total (atmosphere plus ocean) material entropy production, the difference between all simulations is small (ranging from 0.028 to $0.031 \mathrm{~W} \mathrm{~m}^{-2} \mathrm{~K}^{-1}$ ) but still a maximum may be inferred for $k_{\text {oce }}=10^{4} \mathrm{~m}^{2} \mathrm{~s}^{-1}$ (having about the same value as for $k_{\text {oce }}=$ $\left.10^{3} \mathrm{~m}^{2} \mathrm{~s}^{-1}\right)$. Note, however, that the total entropy production (given by the export by long-wave emission and the solar input; see eq. 1) attains its maximum at $k_{\text {oce }}=10^{7} \mathrm{~m}^{2} \mathrm{~s}^{-1}$, that is, the value for $k_{\text {oce }}=10^{4} \mathrm{~m}^{2} \mathrm{~s}^{-1}$ lies in the middle of all simulations.

Summarizing, the entropy production depends sensitively on the oceanic heat transport, whereas the transport itself (parametrized by diffusion) does not provide a significant contribution. The changes of entropy production are small despite the rather drastic changes of the climate. For the atmospheric material entropy production, a MEP can be found for a simulation that is close to the present day climate. This maximum is also present for the total material entropy production (but much 

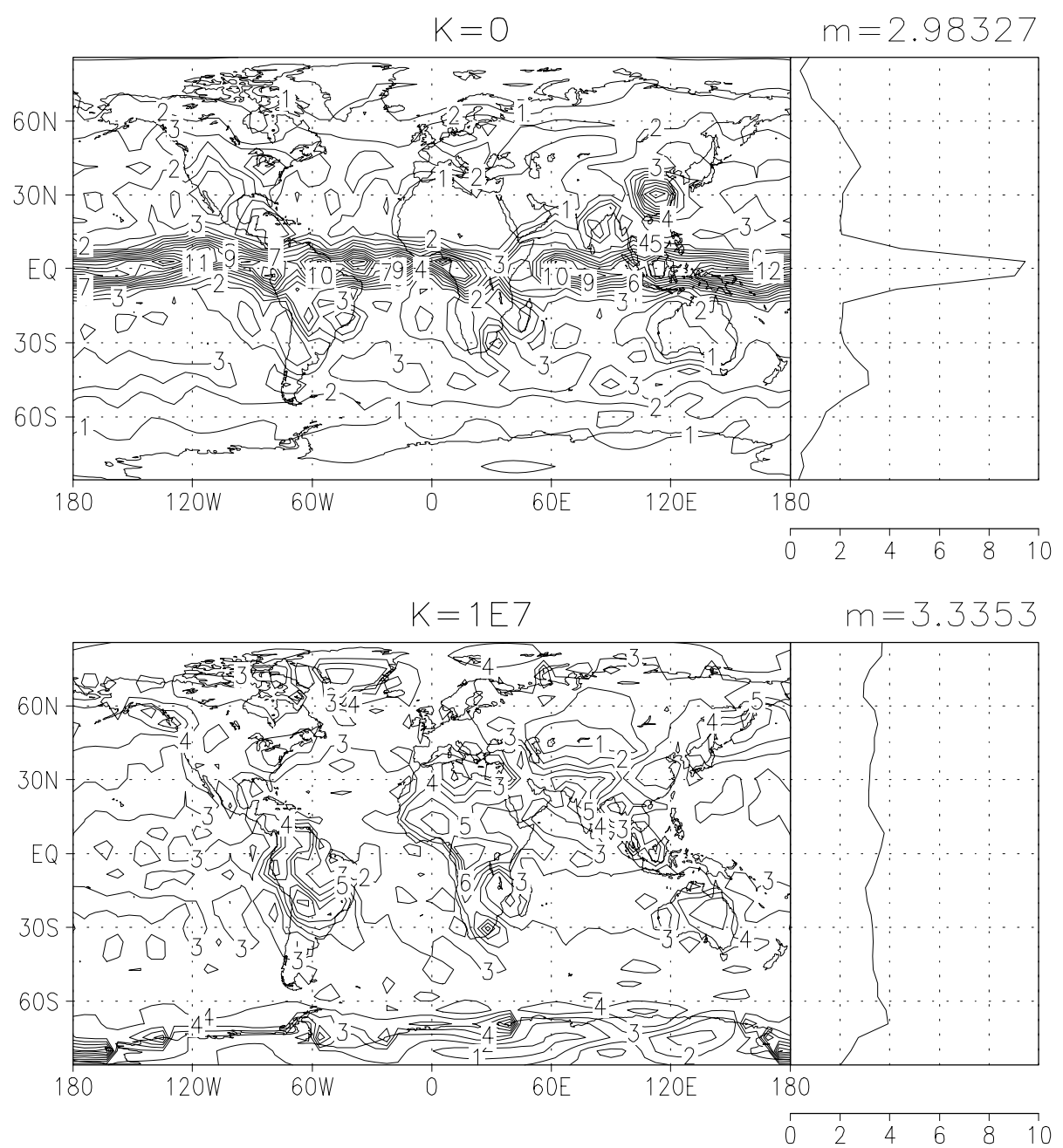

Fig. 5. Horizontal distributions and zonal averages of the annual mean precipitation (in $\mathrm{mm} \mathrm{d}^{-1}$ ) for $k_{\text {oce }}=0$ (top panel) and $k_{\text {oce }}=10^{7}$ (bottom panel).

less pronounced) but cannot be found in the total production (all processes). These results hint to a possible applicability of the MEP principle for the material production in climate models of higher complexity, but it should be kept in mind that the absolute changes of the material entropy production are very small and comparable to the uncertainty of the measure.

\section{Conclusions}

The Planet Simulator, a climate model of intermediate complexity, is used to investigate the entropy balance of the Earth's climate system. Direct computations are compared with the indirect estimates commonly used to assess the entropy production from observations. It is shown that, in the model, the indirectly estimated entropy production by absorption of short-wave radiation and by long-wave emission are in good agreement with the directly computed value, although simplifications and assumptions enter. Large differences (40\%), however, occur for the material entropy production, which plays the key role in entropy production maximization (MEP). In addition, it appears that within the atmosphere, the level-to-level interaction of longwave radiation, which is not considered in the estimates, is more important than the long-wave atmosphere-surface interplay. The level-to-level interaction by long-wave radiation acts as a process similar to diffusion from high to low temperatures. Using 'effective' temperatures obtained from the model together with observed fluxes, yield a modified estimate of the entropy production in the real climate system. As in the model, the material entropy production is significantly lower accounting for $2 \%$ of the total entropy production only.

Sensitivity studies show that even in a drastically changed mean climate (in terms of vegetation cover, oceanic heat transport, atmospheric circulation), the changes of entropy production are small. The changing horizontal diffusivity of the ocean hints to MEP for a climate close to the present day simulation, when considering the atmospheric material contribution to the entropy 
Table 3. Fluxes (in $\mathrm{W} \mathrm{m}^{-2}$ ) and entropy productions (in $\mathrm{W} \mathrm{m}^{-2} \mathrm{~K}^{-1}$ ) for the simulations with different diffusivities $k_{\text {oce }}(\mathrm{a}=$ atmosphere, $\mathrm{s}=$ surface)

\begin{tabular}{|c|c|c|c|c|c|c|}
\hline$k_{\mathrm{oce}}=$ & 0 & $10^{3}$ & $10^{4}$ & $10^{5}$ & $10^{6}$ & $10^{7}$ \\
\hline \multicolumn{7}{|l|}{ Fluxes } \\
\hline Top net solar radiation & 239 & 241 & 244 & 245 & 246 & 246 \\
\hline Outgoing lw radiation & 237 & 239 & 243 & 243 & 244 & 244 \\
\hline Sensible heat flux & 22 & 22 & 23 & 21 & 18 & 17 \\
\hline Latent heat flux & 87 & 90 & 93 & 92 & 95 & 97 \\
\hline \multicolumn{7}{|l|}{ Entropy productions } \\
\hline Absorption of sw radition & $\begin{array}{l}a=0.260 \\
s=0.589\end{array}$ & $\begin{array}{l}a=0.262 \\
s=0.592\end{array}$ & $\begin{array}{l}a=0.265 \\
s=0.597\end{array}$ & $\begin{array}{l}a=0.266 \\
s=0.598\end{array}$ & $\begin{array}{l}a=0.274 \\
s=0.593\end{array}$ & $\begin{array}{l}a=0.277 \\
s=0.591\end{array}$ \\
\hline Emission of lw radiation to space & $\begin{array}{l}\mathrm{a}=-0.757 \\
\mathrm{~s}=-0.157\end{array}$ & $\begin{array}{l}\mathrm{a}=-0.771 \\
\mathrm{~s}=-0.152\end{array}$ & $\begin{array}{l}\mathrm{a}=-0.791 \\
\mathrm{~s}=-0.142\end{array}$ & $\begin{array}{l}\mathrm{a}=-0.795 \\
\mathrm{~s}=-0.138\end{array}$ & $\begin{array}{l}\mathrm{a}=-0.816 \\
\mathrm{~s}=-0.121\end{array}$ & $\begin{array}{l}\mathrm{a}=-0.825 \\
\mathrm{~s}=-0.113\end{array}$ \\
\hline Atm-sfc interaction by lw radiation & $\begin{array}{c}a=0.067 \\
s=-0.061\end{array}$ & $\begin{array}{c}\mathrm{a}=0.070 \\
\mathrm{~s}=-0.063\end{array}$ & $\begin{array}{c}\mathrm{a}=0.077 \\
\mathrm{~s}=-0.070\end{array}$ & $\begin{array}{c}\mathrm{a}=0.087 \\
\mathrm{~s}=-0.080\end{array}$ & $\begin{array}{c}\mathrm{a}=0.099 \\
\mathrm{~s}=-0.091\end{array}$ & $\begin{array}{c}\mathrm{a}=0.102 \\
\mathrm{~s}=-0.094\end{array}$ \\
\hline Internal interaction by $1 w$ radiation & 0.026 & 0.027 & 0.029 & 0.030 & 0.031 & 0.032 \\
\hline Sensible heat flux & $\begin{array}{c}a=0.077 \\
s=-0.075\end{array}$ & $\begin{array}{c}a=0.076 \\
s=-0.074\end{array}$ & $\begin{array}{c}a=0.075 \\
s=-0.072\end{array}$ & $\begin{array}{c}a=0.071 \\
s=-0.069\end{array}$ & $\begin{array}{c}a=0.061 \\
s=-0.060\end{array}$ & $\begin{array}{c}\mathrm{a}=0.058 \\
\mathrm{~s}=-0.056\end{array}$ \\
\hline Latent heat flux & $\begin{array}{c}\mathrm{a}=0.321 \\
\mathrm{~s}=-0.293\end{array}$ & $\begin{array}{c}\mathrm{a}=0.331 \\
\mathrm{~s}=-0.302\end{array}$ & $\begin{array}{c}\mathrm{a}=0.342 \\
\mathrm{~s}=-0.314\end{array}$ & $\begin{array}{c}\mathrm{a}=0.338 \\
\mathrm{~s}=-0.312\end{array}$ & $\begin{array}{c}\mathrm{a}=0.347 \\
\mathrm{~s}=-0.320\end{array}$ & $\begin{array}{c}\mathrm{a}=0.354 \\
\mathrm{~s}=-0.326\end{array}$ \\
\hline
\end{tabular}

production. Note that this maximum is only marginal for the total (atmosphere, land and ocean) material entropy production and absent in the total production (including all processes). These results hint to a possible applicability of the MEP principle for the material production in climate models of higher complexity. It should be kept in mind, however, that the absolute changes of the material entropy production are very small and comparable to the uncertainties in numerics and physics.

The entropy production of the green planet is larger than that of the desert world and the present day climate, which regarding MEP, suggests the green planet to be the most likely climate state. However, in this case constraints like water availability for vegetation may hinder the system to attain this MEP-state. Therefore, in-depth insight into all constraints acting in the climate system is required to achieve evidence of and apply MEP to the observed and simulated climate. In addition, it should be noted that in contrast to the diffusive heat transport in simple models studying MEP (e.g. Paltridge, 1975), the dynamical core of a GCM (which is adiabatic advection) itself does not contribute significantly to the entropy production. Advection (i.e. the meridional transport of heat in a GCM) would, in a perfect numerical set up, conserve entropy. But in the case presented here, numerical dispersion errors and Gibbs oscillations are generated by the spectral approximation, and thus, noise is added to the temperature field, acting as a sink of entropy, which is approximately compensated by artificial horizontal diffusion (as pointed out by Woolings and Thuburn, 2006). In the Planet Simulator, material entropy production is due to the interaction between the atmosphere and the surface by sensible heat flux (parametrizing vertical mixing in connection with the advective transport of sensible heat) and by the conversion of moisture to liquid (after it is transported). Concerning MEP in a GCM this means that, if the MEP principle is valid, it is not the production by the transport process (advection) itself but the production by subsequent processes (here, sensible heat flux and moisture conversion) that is maximized. This maximization will, of course, comprise the optimization of the atmospheric transport to maximize the entropy production by the subsequent processes.

Finally, it is worth noting that the resolution of the model used here is very low. According to Kleidon et al. (2003), the meridional structure has not yet converged to an optimal state (that is, optimal meridional heat transport) at this resolution.

\section{Acknowledgments}

This is part of a review lecture 'Analysing climate models: Longterm memory, entropy, and extremes' presented at the EGU 2007 session 'Climate Model Intercomparison'. Thanks are due to Valerio Lucarini (University of Bologna) for his organization of the session. Helpful comments from two anonymous reviewers are appreciated. K. F. acknowledges financial support by a MaxPlanck fellowship.

\section{References}

Dewar, R. 2003. Information theory explanation of the fluctuation theorem, maximum entropy production and self-organized criticality in non-equilibrium stationary states. J. Phys. A: Math. Gen. 36, 631641.

Eliasen, E., Machenhauer, B. and Rasmussen, E. 1970. On a numerical method for integration of the hydrodynamical equations with a spectral representation of the horizontal fields. Report No. 2, Inst. of Theor. Met., Københavns University, Copenhagen. 
Fraedrich, K., Kleidon, A. and Lunkeit, F. 1999. A green planet versus a desert world: estimating the effect of vegetation extremes on the atmosphere. J. Clim. 12, 3156-3163.

Fraedrich, K., Jansen, H., Kirk, E., Luksch, U. and Lunkeit, F. 2005a. The planet simulator: towards a user friendly model. Meteorol. Zeitschrift 14, 299-304.

Fraedrich, K., Jansen, H., Kirk, E. and Lunkeit, F. 2005b. The planet simulator: green planet and desert world. Meteorol. Zeitschrift 14, 305-314.

Goody, R. 2000. Sources and sinks of climate entropy. Quart. J. Roy. Meteorol. Soc. 126, 1953-1970.

Goody, R. 2007. Maximum entropy production in climate theory. $J$. Atmos. Sci. 64, 2735-2739.

Grassl, H. 1981. The climate at maximum entropy production by meridional atmospheric and oceanic heatfluxes. Quart. J. Roy. Meteorol. Soc. 107, 153-166.

Johnson, D. R. 1997. General coldness of climate models and the second law: implications for modeling the Earth system. J. Clim. 10, 28262846.

Kiehl, J. T. and Trenberth, K. E. 1997. Earth's annual global mean energy budget. Bull. Am. Meteorol. Assoc. 78, 197-208.

Kleidon, A. and Fraedrich, K. 2005. Biotic entropy production and global atmosphere-biosphere interactions. In: Non-Equilibrium Thermodynamics and the Production of Entropy (eds. A. Kleidon and R. D. Lorenz). Springer-Verlag, Berlin, Heidelberg, 173-189.

Kleidon, A., Fraedrich, K. and Heimann, M. 2000. A green planet versus a desert world: estimating the maximum effect of vegetation on the surface energy balance. Clim. Change 44, 471-493.

Kleidon, A., Fraedrich, K., Kunz, T. and Lunkeit, F. 2003. The atmospheric circulation and states of maximum entropy production. Geophys. Res. Let. 30, L18363.

Kleidon, A., Fraedrich, K., Kirk, E. and Lunkeit, F. 2006. Maximum entropy production and the strength of boundary layer exchange in an atmospheric general circulation model. Geophys. Res. Let. 33, L06706.

Kunz, T., Fraedrich, K. and Kirk, E. 2008. Optimisation of simplified GCMs using circulation indices and maximum entropy production. Clim. Dyn. 30, 803-813.

Kuo, H. L. 1965. On formation and intensification of tropical cyclones through latent heat release by cumulus convection. J. Atmos. Sci. 22, $40-63$.

Kuo, H. L. 1974. Further studies of the parameterization of the influence of cumulus convection on large-scale flow. J. Atmos. Sci. 31, 12321240.

Lacis, A. A. and Hansen, K. E. 1974. A parameterization for the absorption of solar radiation in the Earth's atmosphere. J. Atmos. Sci. 31, $118-133$.
Laursen, L. and Eliasen, E. 1989. On the effect of the damping mechanisms in an atmospheric general circulation model. Tellus $\mathbf{4 1 A}$, 385400.

Lettau, H. 1954. A study of the mass, momentum and energy budget of the atmosphere. Archiv für Meteorol. Geophys. Bioklim. 7, 133157.

Lorenz, R. D., Lunine, J. I., Withers, P. G. and MacKay, C. P. 2001. Titan, mars, and earth: entropy production by latitudinal heat transport. Geophys. Res. Let. 28, 415-418.

Louis, J. F. 1979. A parametric model of vertical eddy fluxes in the atmosphere. Bound. Layer Meteorol. 17, 187-202.

Louis, J. F., Tiedke, M. and Geleyn, J.-F. 1982. A short history of the PBL parameterisation at ECMWF. In: Proceedings of the ECMWF Workshop on Planetary Boundary Layer Parameterization. Reading, 25-27 Nov. 1981, 59-80.

Orszag, S. A. 1970. Transform method for calculation of vector coupled sums. J. Atmos. Sci. 27, 890-895.

Ozawa, H., Ohmura, A., Lorenz, R. D. and Pujol, T. 2003. The second law of thermodynamics and the global climate system: a review of the maximum entropy production principle. Rev. Geophys. 41, 1018, 4.1-4.24.

Paltridge, G. W. 1975. Global dynamics and climate-a system of minimum entropy exchange. Quart. J. Roy. Meteorol. Soc. 101, 475484.

Peixoto, J. P. and Oort, A. H. 1992. Physics of Climate. Am. Inst. Phys., 520pp.

Peixoto, J. P., Oort, A. H., de Almeida, M. and Tome, A. 1991. Entropy budget of the atmosphere. J. Geophy. Res. D6, 10 981-10 988.

Roeckner, E., Arpe, K. and Bengtsson, L. 1992. Simulation of presentday climate with the ECHAM model: impact of model physics and resolution. Technical Report 93, Max-Planck-Institut.

Sasamori, T. 1968. The radiative cooling calculation for application to general circulation experiments. J. Appl. Meteorol. 7, 721729.

Slingo, A. and Slingo, J. M. 1991. Response of the national center for atmospheric research community climate model to improvements in the representation of clouds. J. Geophys. Res. 96, 341357.

Stephens, G. L. 1978. Radiation profiles in extended water clouds. II: parameterization schemes. J. Atmos. Sci. 35, 2123-2132.

Stephens, G. L., Ackermann, S. and Smith, E. A. 1984. A shortwave parameterization revised to improve cloud absorption. J. Atmos. Sci. 41, 687-690.

Weiss, W. 1996. The balance of entropy on earth. Cont. Mech. Thermodyn. 8, 37-51.

Woollings T. and Thuburn, J. 2006. Entropy sources in a dynamical core atmosphere model. Quart. J. Roy. Meteorol. Soc. 132, 43-59. 